\title{
Perché la diffusione della dialisi peritoneale è ancora insoddlisfacente
}

\author{
Carlo Rugiu, Antonio Lupo
}

\author{
Cattedra e Divisione di Nefrologia, Ospedale Civile Maggiore, Verona
}

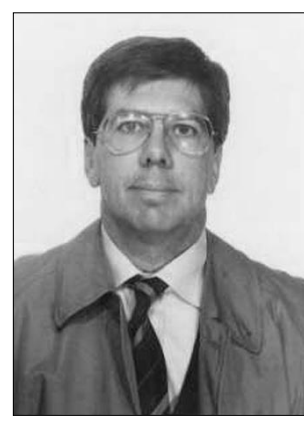

Carlo Rugiu
I in ottenuti in Italia in termini di penetrazione della dialisi peritoneale non possono dirsi soddisfacenti: infatti, nonostante essa sia oramai considerata una valida alternativa alla emodialisi, i valori di incidenza e di prevalenza, rispettivamente pari al 18 e $10 \%$ secondo il RIDT aggiornato al 31.12.1998, la penalizzano fortemente e ne confermano il ruolo di attore non protagonista sulla scena del trattamento sostituivo della funzione renale in Italia. In realtà, questa prevalenza del $10 \%$ è un valore medio che scaturisce da due diverse, ben distinte, realtà nefrologiche.

Infatti, mentre in alcune regioni, caratterizzate da una Sanità pressocché completamente pubblica, la prevalenza della dialisi peritoneale si colloca fra il 10 e il $20 \%$, in altre, localizzate per lo più, ma non esclusivamente al Centro-Sud, dove i Centri di dialisi pubblici sono una minoranza, la prevalenza della dialisi peritoneale resta ben al di sotto del 5-6\%, su percentuali del tutto marginali. Questa è una situazione nota da tempo, che trova una sua spiegazione in una maggiore presenza della Sanità privata nel settore della dialisi: ovviamente, vi sono interessi economici che facilitano e perpetuano questa situazione, ma sarebbe semplicistico e riduttivo imputare ai minori (talvolta minimi) rimborsi previsti per la dialisi peritoneale la causa principale della sua "marginalità".

In realtà, quello che ancora oggi ci deve preoccupare e sul quale dobbiamo interrogarci è perché dove le condizioni "ambientali" sono favorevoli (regioni dell'Italia settentrionale) la penetrazione della dialisi peritoneale resti così modesta.

A nostro avviso i principali fattori responsabili di tale situazione sono:

- la coscienza collettiva;

- i pazienti "late-referral";

- il variare delle condizioni clinicofamiliari-sociali;

- la "forma mentis" del nefrologo.

Con il termine di coscienza collettiva, ci riferiamo a quella immagine ben radicata, non solo su rotocalchi e mass media, ma anche all'interno del mondo medico, talvolta anche fra $\mathrm{i}$ nefrologi, per la quale quando si parla di dialisi ci riferisce automaticamente alla emodialisi. Quest'ultima, sia pure forte di un "vantaggio" di circa venti anni sulla peritoneale, costituisce nella coscienza collettiva l'unica forma di terapia sostitutiva della funzione renale, mentre quando si parla di dialisi peritoneale bisogna sempre iniziare spiegando "che cos'è il peritoneo".

Un altro problema che può in parte spiegare la difficile crescita della dialisi peritoneale è quello dei pazienti "late-referral": soprattutto in passato, i pazienti che giungevano per la prima volta in una Divisione nefrologica già uremici iniziavano il trattamento sostitutivo d'urgenza in emodialisi tramite un accesso vascolare estemporaneo, a questa tendevano ad "affezionarsi" ed erano poi riluttanti a staccarsene. In quest'otti$\mathrm{ca}$, risultava più difficile proporgli sullo stesso piano una metodica quella peritoneale - che in un primo tempo era stata posposta per motivi di emergenza clinica o di carattere organizzativo. È quindi auspicabile una più fattiva collaborazione fra il nefrologo e potenziali "serbatoi" di uremici, quali la medicina di base e i Centri antidiabetici, per la crescente incidenza di nefropatia diabetica e di nefropatie nel paziente diabetico, al fine di limitare il numero dei "late-referral" e di pianificare per tempo, con una informazione multidisciplinare serena, bilanciata e personalizzata la scelta della metodica dialitica.

Peraltro, nella pratica clinica, si possono presentare alcune situazioni, 
quali improvvise variazioni dell'assetto familiare (morte del coniuge= perdita del potenziale partner, un elemento di crescente rilevanza e importanza sul piano organizzativo e sociale), inattese complicanze cliniche (interventi chirurgici, accidenti cerebrovascolari, deterioramento delle funzioni cognitive), modificazioni del vissuto del paziente (esperienze negative a contatto con dializzati peritoneali) che possono vanificare 1'opera di "preparazione del terreno" e di corretta informazione da parte di una équipe multidisciplinare, e shiftare così verso l'emodialisi un possibile, valido candidato alla dialisi peritoneale.

Infine, vi è da considerare il ruolo della "forma mentis" del nefrologo verso la metodica dialitica: all'inizio degli anni '80 la dialisi peritoneale era considerata ancora "in gestazione" rispetto alla emodialisi ed era spesso riservata a pazienti critici o con minor aspettativa di vita; anche questo atteggiamento, frequentemente sostenuto dai medici che non si occupavano specificamente di dialisi peritoneale, contribuì alla sua minore diffusione.

A distanza di circa vent'anni, le nuove generazioni di nefrologi, che si sono formate una coscienza dialitica imparziale a contatto con una dialisi peritoneale "adulta" in grado di offrire sistemi di connessione sicuri, sacche in materiali biocompatibili, agenti osmotici alternativi al glucosio, metodiche automatizzate in grado di dializzare e riabilitare adeguatamente il paziente potranno rendere possibile quel salto di quantità, ovvero una maggiore diffusione, della dialisi peritoneale che noi tutti ci auguriamo.

carlo.rugiu@mail.azosp.vr.it 